\title{
Local phase measurements of light in a one-dimensional photonic crystal
}

\author{
E. FLÜCK, A. M. OTTER, J. P. KORTERIK, M. L. M. BALISTRERI, L. KUIPERS \& \\ N. F. VAN HULST \\ Applied Optics Group, Department of Applied Physics and MESA ${ }^{+}$Research Institute, \\ University of Twente, PO Box 217, 7500 AE Enschede, The Netherlands
}

Key words. Coherent, light scattering, near-field optics, photonic crystals, scanning probe microscopy.

\section{Summary}

For the first time the local optical phase evolution in and around a small, one-dimensional photonic crystal has been visualized with a heterodyne interferometric photon scanning tunnelling microscope. The measurements show an exponential decay of the optical intensity inside the crystal, which consists of a periodic array of subwavelength air rods fabricated in a conventional ridge waveguide. In addition it is found that the introduction of the air rods has a counterintuitive effect on the phase development inside the structure. The heterodyne detection scheme allows the detection of low-intensity scattered waves. In the vicinity of the scattering air rods phase singularities are found with a topological charge of plus or minus one.

\section{Introduction and motivation}

Fabricating and understanding light scattering and propagation on scales approaching the wavelength of light itself has received significantly increased attention in the last few years. An important reason for the increase is the advent of a new kind of optical material: the photonic crystal (Soukoulis, 1996). In these crystals, of which the index of refraction is varied periodically, the dispersion relations of photons can be affected even to the extent that ranges of forbidden frequencies, band gaps, open up. As a result, careful tailoring of the crystal structure by deliberate introduction of defects allows the flow of light to be molded efficiently. Thus, it is anticipated that the photonic crystals will play a crucial role in telecommunication in the (near) future. Essential ingredients for the crystals are multiple scattering of light by the subwavelength index variations and a strong interaction between geometry on the one hand and optical properties on the other (Vos et al., 1996). The

Correspondence to: E. Flück. Tel.: +31 53489 3871; fax: +31 53489 1105; e-mail: e.flueck@tn.utwente.nl control of fabrication steps and their consequences for the optical properties of the material require accurate investigation tools. As the effect of local variations in geometry on light propagation can be profound, an instrument that probes local optical and geometrical properties simultaneously is of the essence. A photon scanning tunnelling microscope (PSTM) is such a tool. Recently, several investigations on optical intensity distributions in complex photonic structures have been reported (Choo et al., 1995; Jackson et al., 1995; Vander Rhodes et al., 1998; Balistreri et al., 1999; Krenn et al., 1999; Phillips et al., 1999; Mulin et al., 2000; Peeters et al., 2000). However, measurements of the optical phase development inside such structures have, as yet, never been reported. Vaez-Iravani \& ToledoCrow (1993) showed that the optical phase can be measured in a near-field microscope by means of pseudoheterodyne detection. They demonstrated the detection scheme on simple model structures.

Here, we present the first ever measurements on tailormade one-dimensional crystals with a heterodyne interferometric PSTM. Not only optical intensity distributions and topography, but also the phase evolution of the light in these advanced photonic structures can be measured by this instrument. The wavelength-dependent measurements reveal a rich variety of optical phenomena: scattering, exponential damping and the development of phase singularities.

\section{Materials and methods}

In a conventional PSTM the intensity distribution of light in a structure is measured simultaneously with the topography of the structure. A tapered fibre probe, kept at a constant height above the sample surface with shear-force feedback, frustrates the evanescent field above the structure. As a result, a propagating guided mode develops in the fibre, which is subsequently detected. As the probe is raster scanned across the surface a map of the intensity 
distribution inside the structure is constructed. Peeters et al. (2000) found that the use of coated aperture probes is crucial for the investigation of subwavelength scatterers in order to minimize the amount of light scattered directly into the probe. Our probes have been fabricated by a combination of fibre pulling, Al coating and side-on milling with a focused ion beam, as described by Veerman et al. (1998).

In order to visualize the phase evolution in the structure, we have modified our conventional PSTM in the following way (Balistreri et al., 2000). The PSTM has been incorporated in one leg of a Mach-Zehnder interferometer. To this end, the laser light is split in two parts. One part is coupled into the photonic structure and is subsequently picked up by the PSTM. The second part forms the reference branch. Both branches are then recombined and allowed to interfere. The interference allows the phase inside the structure to be determined, as the optical pathlength of the signal branch only changes by the distance (times the index of refraction of the sample) over which the probe is scanned along the sample. By means of an acousto-optic modulator the optical frequency of the reference branch is shifted by $40 \mathrm{kHz}$ in order to allow heterodyne detection of the interference signal. Subsequent detection with a lock-in amplifier produces two signals: one proportional to the optical amplitude times the cosine of the phase and the second proportional to the amplitude times the sine of the phase. Simple mathematics then suffices to determine the phase and the amplitude separately. In one measurement a twodimensional map of the topography is obtained simultaneously with the optical field distribution containing both the amplitude and the phase information. Before each measurement, the stability and drift of the phase measurement is checked, as phase detection is very sensitive to any air flow or temperature variation. To this end, a line scan along the waveguide axis in time is performed before and after each measurement to observe any change in position of the phase pattern.

The basis for our photonic crystals is formed by a $\mathrm{Si}_{3} \mathrm{~N}_{4}$ ridge waveguide. On top of a $3 \mu \mathrm{m} \mathrm{SiO}{ }_{2}$ layer, $55 \mathrm{~nm} \mathrm{Si}_{3} \mathrm{~N}_{4}$ is grown by low-pressure chemical-vapour deposition. Then a waveguide ridge of $11 \mathrm{~nm}$ height and $1.4 \mu \mathrm{m}$ width is etched by reactive-ion etching. For the wavelength used in this investigation only the fundamental waveguide mode for transverse electric (TE) polarized light (E-field in the plane of the waveguide) is supported by the waveguide. With the effective index method (EIM) of Tamir (1975), the effective refractive index of our structure is calculated for each wavelength used. The photonic crystals are fabricated in the ridge waveguide by means of a focused ion beam. With this instrument we can mill subwavelength holes of arbitrary shape with dimensions down to approximately $30 \mathrm{~nm}$. Here, we have fabricated a periodic array of 15 air rods with periodicity of $220 \mathrm{~nm}$ and an air rod diameter of $110 \mathrm{~nm}$. The spacing between the holes and the size of the holes has an accuracy better than $10 \mathrm{~nm}$. The range of wavelengths used in the experiment was 585-647 $\mathrm{nm}$. To this end, a variety of laser sources was employed: a $\mathrm{He}-\mathrm{Ne}$ laser, an $\mathrm{Ar}-\mathrm{Kr}$ laser and a frequency-doubled, Ti: sapphire-pumped optical parametric oscillator.

\section{Experimental results}

Light is coupled into the waveguide such that the fundamental TE-mode is excited. Figure 1 shows the simultaneously obtained topography (a), field intensity (b) and cosine of the phase (c) as obtained with the heterodyne interferometric PSTM. The waveguide ridge and the 15 air rods of $110 \mathrm{~nm}$ diameter are clearly visible in Fig. 1(a). The lateral resolution in the topography is found to be $50 \mathrm{~nm}$. Note that a high position accuracy of the probe $(0.4 \mathrm{~nm})$ is achieved through the use of a position feedback scheme. The light in the measurements propagates from top to bottom. In the upper half of Fig. 1(b) a periodic pattern with horizontal stripes is visible. The origin of those horizontal stripes is interference between incoming light and light that is backscattered from the air rods. Thus, a standing wave is built up in front of the periodic structure. The wavelength of the guided mode and thus the effective index of the waveguide can be determined from the periodicity of the standing wave. In the vicinity of the periodic air rod array, we observe that light is able to travel on the left and right side along the structure. The intensity inside the air rod region decays rapidly. The pattern in Fig. 1(c) is dominated by the straight wavefronts, corresponding to a plane wave propagating from top to bottom. The length of the wavefront periods that corresponds directly to the wavelength of light propagating in the structure is, as expected, exactly twice that of the standing waves observed in the image of the optical intensity (Fig. 1c). Information of the standing wave is also present in the phase map. However, as only a small fraction (see below) of the incoming light is backreflected from the air rods, the pattern is dominated by a period corresponding to the wavelength of the incoming light. Close examination of the centre part of Fig. 1(c) (indicated by the dashed oval), shows small variations in the otherwise straight phase front lines. This shows the effect of the introduction of the air rods on the local evolution of the optical phase as light propagates through the photonic crystal area.

Figure 2 depicts a line trace through the intensity image, along the waveguide axis (Fig. 1b, dashed line). A high intensity peak is located directly at the beginning of the periodic air rod array. It is clear that the optical intensity decreases both into the structure and in the backscattered direction. As discussed by Peeters et al. (2000), the decay of the intensity of the standing wave in front of the air rod array can be explained in terms of the excitation via the scattering process of so-called leaky modes in addition to 

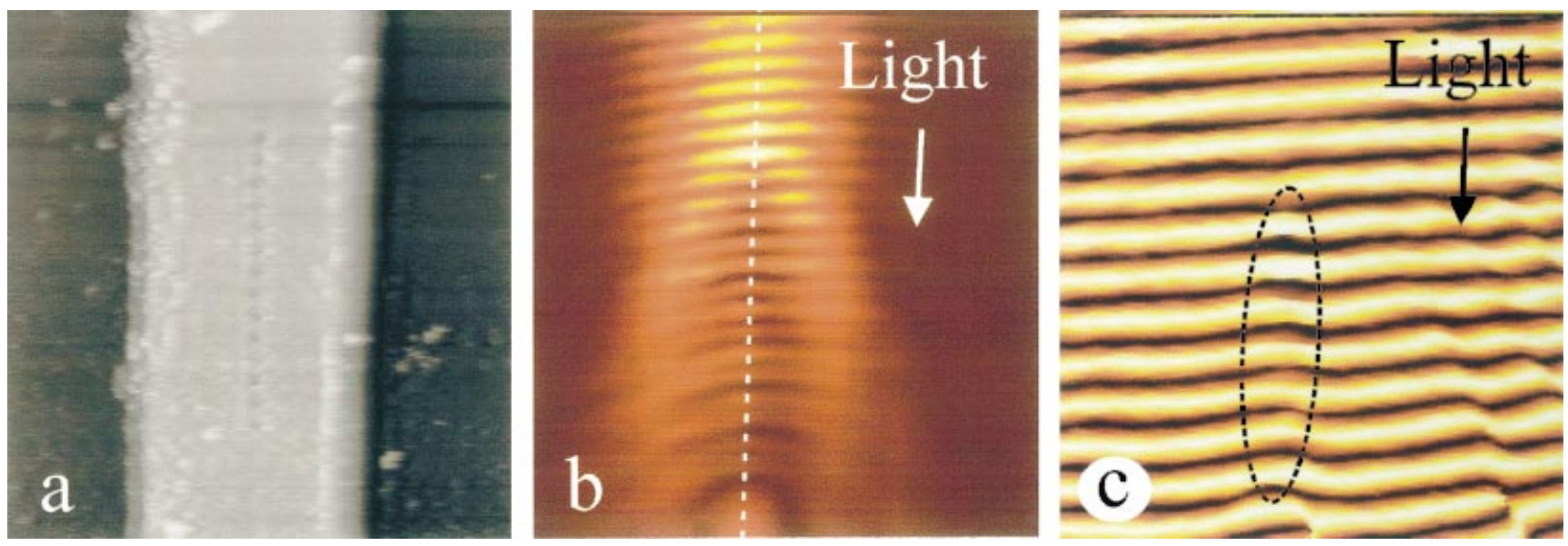

Fig. 1. Interferometric PSTM measurement of a $\mathrm{Si}_{3} \mathrm{~N}_{4}$ ridge waveguide containing a small, one-dimensional photonic crystal consisting of a periodic linear array of subwavelength air rods $\left(3.97 \times 5.34 \mu^{2}\right)$. (a) Topography image in which the air rods with a diameter of $110 \mathrm{~nm}$ are clearly resolved. A slight double-tip artefact is also visible. The artefact does not seem to have a pronounced influence on the optical measurements. (b) Intensity distribution. The arrow indicates the direction of the incoming light $(\lambda=632.8 \mathrm{~nm}$ in air). A bright horizontal intensity pattern above the air rod array is visible. Interference between incoming and backreflected light results in a standing wave. At the location of the array, the intensity is clearly reduced, while some light seems to travel along either side of the air rods. The dashed line indicates the position at which the line trace (Fig. 2) is taken. (c) Map of the cosine of the phase in the structure. The image is dominated by horizontal wavefronts, indicating that, on the whole, light is propagating as a plane wave from top to bottom. In the region of the air rod array (dashed oval) a clear distortion of the wavefronts is observed, which indicates that the local index of refraction is modified by the introduction of the air rods. Surprisingly, a first glance suggests that the index has locally increased with respect to that of the waveguide. In the right bottom of the image phase jumps are visible outside the ridge waveguide.

the fundamental guided mode of the waveguide. In addition, circular propagating waves (see Fig. 3) excited due to scattering in the periodic crystal region may contribute as well to the interference pattern in front of the periodic air rod array. These circularly shaped waves will also decrease in intensity with increasing distance from the air rods. The periodicity of the standing wave allows us to determine the wavelength of the light in the waveguide and hence also the effective index of refraction of the waveguide. The experimentally determined values were then compared to wavelengths calculated with the EIM. The decay of the optical intensity inside the photonic crystal is quantified by fitting an exponential decay $\mathrm{e}^{-\beta \mathrm{x}}$ (as shown in Fig. 2) through the intensity maxima found in the air rod region. The reflectivity of the air rod array can be determined from the modulation depth, $M=\left(I_{\max }-I_{\min }\right) /$ $\left(I_{\max }+I_{\min }\right)$, of the standing wave, where $I_{\max }$ and $I_{\min }$ are the intensity of the maxima and the minima, respectively. Then the amplitude reflection coefficient is calculated using $R=\left(1+\sqrt{ }\left(1-M^{*} M\right)\right) / M$. This quantitative analysis was performed for different wavelengths of the in-coupled light (range $585-647 \mathrm{~nm}$ ). The results of this analysis are summarized in Table 1.

It is obvious that the experimental and the calculated values for the wavelengths show excellent agreement. A clear wavelength dependence of the decay rate inside the array of scatterers is not apparent. It does seem that the

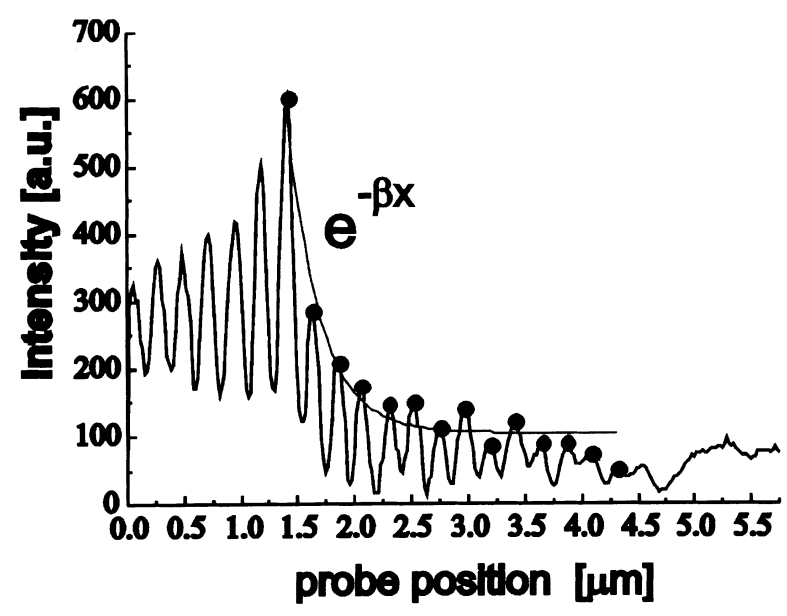

Fig. 2. A line trace through the intensity distribution of Fig. 1(b) (at the dashed line). The direction of the incoming light is from left to right. A periodic intensity distribution is visible with a periodicity corresponding to half the incoming wavelength, which indicates that a standing wave has formed. At the location of the first air rod a high intensity peak is observed. The intensity falls off to the left and the right. The decrease in intensity to the left is attributed to the high loss rates of the leaky modes excited in the waveguide. The wavelength dependence of the decrease to the right suggests that it is probably attributable to scattering at each individual air rod. The fluent curve is the best fit of an exponential decay to the solid dots. 


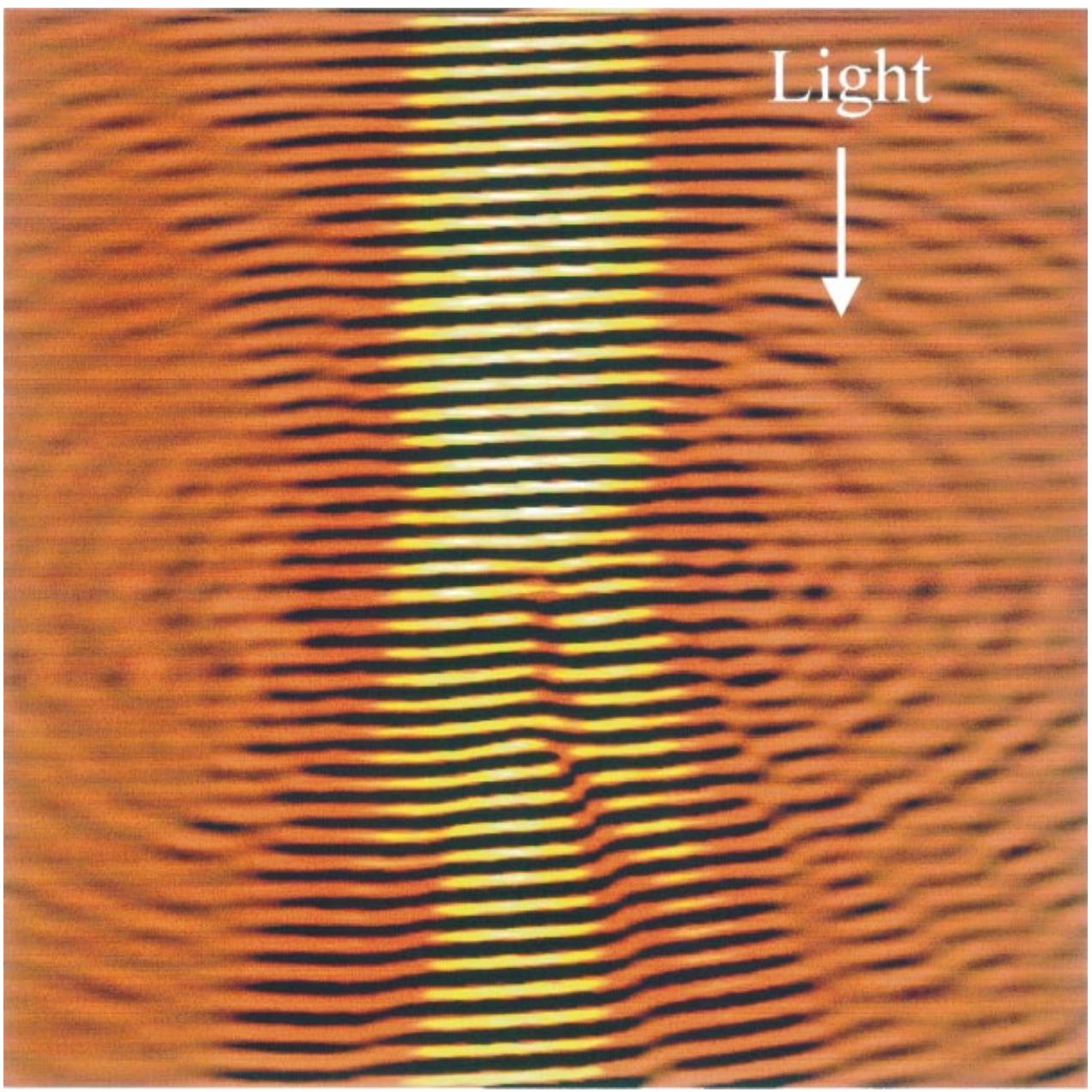

Fig. 3. A zoomed-out interference PSTM measurement of the same area as is depicted in Fig. $1\left(9.37 \times 14.25 \mu \mathrm{m}^{2}\right)$. Here, the amplitude times the cosine of the phase is depicted. In addition to the observation made in Fig. 1, circular waves are observed to originate from the scattering region. It is also apparent that after the decay in amplitude at the location of the photonic crystal, the guided mode of the waveguide 'recovers'. Two 'rays' of light leave this area under an angle of approximately $20^{\circ}$ with the incoming wavevector. Just below (to the right) of the air rod array a network of phase singularities can be seen.

decay rate increases (smaller $1 / \beta)$ with decreasing wavelength. Measurements on waveguides containing a periodic array of 15 slits instead of holes (period $220 \mathrm{~nm}$ ), show this relation between exponential decay and wavelength much more clearly. The reflectivity of the air rods is found in the order of $10 \%$. No clear variation of reflectivity with wavelength is observed. If anything, the reflectivity increases with decreasing wavelength. Unfortunately, we find no evidence for the appearance of a stop gap of the photonic crystal.

With respect to the phase information we have so far concentrated on effects close to the subwavelength scatterers. In order to visualize the full range of scattering phenomena that occur, Fig. 3 shows the product of the optical amplitude and the cosine of the phase for a scan area of $9.37 \mu \mathrm{m} \times 14.25 \mu \mathrm{m}$. Again, light travels from top of the image inside the waveguide (centre of image). The periodic air rod array is located in the middle of the image. A period corresponding to the wavelength $(416 \pm 11 \mathrm{~nm})$ is clearly visible in the top of the image. At the position of the air rods the signal decreases rapidly but light is seen to propagate on either side of the scatterers (see also Fig. 1b). Just behind the subwavelength scatterers, a guided single mode can be seen to recover. As expected, the mode behind the scatterers is found to have the same wavelength as the mode in front of the scatterers. Surprisingly, the wavelength inside the air rod array is found to be smaller than the wavelength inside the ridge waveguide itself (see also Fig. 1c). This presents us with an, as yet unsolved, puzzle as the introduction of air rods would suggest that the index of refraction becomes smaller, corresponding to a larger wavelength. Several possible explanations present themselves. Firstly, a topographical artefact could introduce apparent phase variations. However, as the period of the air rods is on 
Table 1. Results of quantitative analysis of wavelength dependent interferometric PSTM measurements. For different wavelength of the in-coupled light (column 1), the period of the standing wave (column 2) in front of the photonic crystal is determined. The values are in good agreement with calculations (column 3). The wavelength dependency of the exponential intensity decay inside the air rod region (column 4). Column 5 gives the amplitude reflection coefficient as calculated from the measured modulation depth of the standing waves in front of the air rods.

\begin{tabular}{llccr}
$\begin{array}{l}\text { Wavelength } \\
(\mathrm{nm})\end{array}$ & $\begin{array}{c}\text { Period }(\mathrm{nm}) \\
\text { (experiment) }\end{array}$ & $\begin{array}{l}\text { Period }(\mathrm{nm}) \\
(\text { calculated })\end{array}$ & $1 / \beta(\mu \mathrm{m})$ & $R(\%)$ \\
\hline 585 & $198.8(2.4)$ & 199.5 & 0.69 & 13 \\
600 & $202.5(1.9)$ & 205.0 & 0.58 & 10 \\
610 & $208.2(2.0)$ & 208.5 & 0.61 & 2 \\
615 & $212.8(16.4)$ & 210.3 & 0.73 & 6 \\
633 & $214.1(3.8)$ & 216.7 & 0.35 & 11 \\
647 & $220.3(0.9)$ & 221.7 & 0.84 & 8 \\
\hline
\end{tabular}

the order of half the wavelength of the light in the structure, we would expect a 'high frequency' modulation of the phase signal rather than an apparently smaller wavelength. In the near future we will investigate possible topographical artefacts by also measuring at constant heights rather than with a constant probe-surface separation. Secondly, the periodicity of the refractive index can have a profound effect on the optical dispersion relation of light (Soukoulis, 1996). As a result, the effective index of the material can be higher than the introduction of a certain volume fraction of air would suggest; however, never to the extent that the index would become higher than that of the host material. Thirdly, in the area of the air rods the effective index experienced by the light, which is actually picked up by the PSTM probe, might be dominated more by the thin top layer of $\mathrm{Si}_{3} \mathrm{~N}_{4}$ (refractive index 2.01). In the rest of the waveguide the lower index of the $\mathrm{SiO}_{2}$ layer actually dominates the effective index of refraction experienced by the guided mode. Attractive as the third explanation may seem, it is not clear to us whether such a process is actually possible.

Figure 3 also shows two 'rays' of light emerging from the scattering region at angles of plus and minus $20^{\circ}$ with respect to the incoming wavevector. As a result of the increased sensitivity of the heterodyne detection scheme with respect to conventional PSTM schemes, we also observe roughly circular waves of low intensity scattering away from the air rods. Many phase singularities (Balistreri et al., 2000) can be observed: these are positions where a wavefront terminates. Integrating the phase around such a point will result in an accumulated phase of $N \times 2 \pi$, where $N$ is the so-called topological charge of the singularity ( $N= \pm 1$ in these measurements). Note that only the phase singularities in the bottom-right half of the image close to the scatterers are true phase singularities in that their position is fixed and the local optical amplitude is zero. Simple calculations to understand the complex pattern of the cosine of the phase have been performed. The interference between the field of a number of point sources and an incoming mode in a waveguide are compared with Fig. 3. These model calculations show that images like Fig. 3 contain a wealth of information by which the scattering due to ordered arrays of subwavelength scatterers can be better understood.

\section{Conclusions}

For the first time, measurements with a heterodyne interferometric PSTM on light inside small, one-dimensional photonic crystals have been performed with subwavelength resolution. The novel technique allows full characterization of local optical phenomena in the structure under scrutiny. An exponential decay of intensity inside the periodic array is measured which seems to be wavelength-dependent. The effect of local variations of the refractive index due to the introduction of the air rods has been visualized in the phase images. As a result of the increased sensitivity due to the heterodyne detection scheme, low-intensity circular waves with their origin in the scattering region are observed. Phase singularities in the vicinity of the air rods are also revealed.

\section{Acknowledgements}

This work is part of the research programme of the Stichting for Fundamenteel Onderzoek der Materie (FOM), which is financially supported by the Nederlandse Organisatie voor Wetenschappelijk Onderzoek. The research is also part of the Strategic Research Orientation on Advanced Photonic Structures.

\section{References}

Balistreri, M.L.M., Klunder, D.J.W., Blom, F.C., Driessen, A., Hoekstra, H.W.J.M., Korterik, J.P., Kuipers, L. \& van Hulst, N.F. (1999) Visualizing the whispering gallery modes in a cylindrical optical microcavity. Opt. Lett. 24, 1829-1831.

Balistreri, M.L.M., Korterik, J.P., Kuipers, L. \& van Hulst, N.F. (2000) Local observation of phase singularities in optical fields in waveguide structures. Phys. Rev. Lett. 85, 294-297.

Choo, A.G., Chudgar, M.H., Jackson, H.E., De Brabander, G.N., Kumar, M. \& Boyd, J.T. (1995) Photon scanning-tunnelingmicroscopy of optical channel wave-guides. Ultramicroscopy, 57, $124-129$.

Jackson, H.E., Lindsay, S.M., Poweleit, C.D., Naghski, D.H., De Brabander, G.N. \& Boyd, J.T. (1995) Near field measurements of optical channel waveguide structures. Ultramicroscopy, 61, 295298.

Krenn, J.R., Dereux, A., Weeber, J.C., Bourillot, E., Lacroute, Y., Gourdonnet, J.P., Schider, G., Gotschy, W., Leitner, A., Aussenegg, 
F.R. \& Girard, C. (1999) Squeezing the optical near-field zone by plasmon coupling of metallic nanoparticles. Phys. Rev. Lett. 82, 2590-2593.

Mulin, D., Spajer, M., Courjon, D., Carcenac, F. \& Chen, Y. (2000) Near-field probing control of optical propagation in bidimensional guiding mesostructures. J. Appl. Phys. 87, 534-537.

Peeters, C., Flück, E., Otter, A.M., Balistreri, M.L.M., Koerterik, J.P., Kuipers, L. \& van Hulst, N.F. (2000) Photon scanning tunneling microscopy of tailor-made photonic structures. Appl. Phys. Lett. 77, 142-144.

Phillips, P.L., Knight, J.C., Mangan, B.J., Russell, P., St. J., Charlton, M.D.B. \& Parker, G.J. (1999) Near-field optical microscopy of thin photonic crystal films. J. Appl. Phys. 85, 6337-6342.

Soukoulis, C.M. (1996) Photonic Band Gap Materials. Kluwer Verlag, Dordrecht.

Tamir, T. (1975) Integrated Optics. Springer Verlag, Berlin.
Vaez-Iravani, M. \& Toledo-Crow, R. (1993) Phase contrast and amplitude pseudoheterodyne interference near field scanning optical microscopy. Appl. Phys. Lett. 62, 1044-1046.

Vander Rhodes, G.H., Ünlü, M.S., Goldberg, B.B., Pomeroy, J.M. \& Krauss, T.F. (1998) Characterisation of waveguide microcavities using high-resolution transmission spectroscopy and near-field scanning optical microscopy. IEE Proc. Optoelectron. 145, 379383.

Veerman, J.A., Otter, A.M., Kuipers, L. \& van Hulst, N.F. (1998) High definition aperture probes for near-field optical microscopy fabricated by focused ion beam milling. Appl. Phys. Lett. 72, 3115-3117.

Vos, W.L., Sprik, R., van Blaaderen, A., Imhof, A., Lagendijk, A. \& Wegdam, G.H. (1996) Strong effects of photonic band structures on the diffraction of colloidal crystals. Phys. Rev. B, 53, 1623116235. 